\title{
Analysing Mental Health Evaluation Techniques from a Statistical Perspective
}

\author{
Sushma Koushik $\mathrm{N}^{1, *}$, Deepu $\mathrm{R}^{2}$ \\ 1,2 Department of Computer Science \& Engineering, Maharaja Institute of Technology Mysore, India \\ "Corresponding author.Email: sushmak_cse@mitmysore.in
}

\begin{abstract}
Mental wellbeing is an epitome of success for human beings. Recently, a lot of mental diseases that include anxiety, suicidal tendency, depression, bipolar disorder, obsessive compulsive disorder (OCD), etc. have deep-seated among all age groups. This is due to the fact that the social complexity has increased at work, in relationships, at home and in majorly all aspects of human life. Early detection of these problems can help in treating and if possible, eradicating this illness from a person's life. Researchers have proposed different techniques that can be put into action to effectively identify these diseases. These techniques utilize different activities of normal day-to-day human behaviour, like speech analysis, social media behavioural patterns, visual-activity pattern analysis, etc. Due to a large variety of available techniques, researchers find it difficult to standardize techniques for detection and evaluation of such diseases. Thus, in this paper we have performed an in-depth study about the state-of-the art methods to identify mental health issues. Readers will be able to get a bird's eye view of these methods and will be able to identify the best practices involved in mental health analysis. Moreover, this survey also recommends some possible improvements in the existing methods, in order to further improve the system's performance.
\end{abstract}

Keywords: Anxiety, Depression, Mental Health, Social Media, Speech Analysis, Suicidal Tendency.

\section{INTRODUCTION}

In order to evaluate the mental health of the patient, doctors run a series of tests. These tests process the input data from patients, and produce results based on historic data. These inputs come from a wide variety of sources, that include but are not limited to,

- Speech patterns when patient is subjected to different kind of questions.

- General and specific EEG patterns of the patient.

- General and specific ECG patterns of the patient.

- Day to day social media interactions of the patient.

- Body parameters of the patient during doctor sessions.

- Visual behaviour of the patient when subjected to stress-like situations.

Apart from these inputs, doctors and medical examiner can get other historic data for the patient like family history, previous incidences in life, etc. helping to frame a picture about the patient's current and future mental status. They

(Doctors and health analysts), collect the data from different patients forming training corpus, generally consists of the patient's symptoms and their previous mental conditions. The accuracy with which this training corpus is collected, decides the accuracy of future detection of patient's mental health. Health examiners usually take help from software designers to develop systems that take these parameters as input and produce approximate mental health condition of the patient. These software systems are designed using the following steps,

- Dataset selection

- Effective feature extraction

- Feature selection

- Classification

- Post-processing

Dataset selection is characterized by identifying patients who are most suited for the task of classification. These patients must vary in terms of disease type, age, gender, geography, etc. Each patient type must have multiple input features varying in terms of the given factors. In doing so, a stronger dataset will be selected, thereby a stronger classification model will be created. The data from these patients is given to a feature extraction stage, wherein different features from this input data are extracted. Feature selection process is applied to the best \& most varying features for the given dataset. Better features mean a higher chance of proper classification. Algorithms like variance-based methods, key-point selection methods, etc. are used in order to find the optimum feature set. Once the features are evaluated, then a classifier is applied in order to obtain the final category of disease for the patient. These classifiers generally use a feedback mechanism like the one used by backpropagation neural networks, to learn from their results and obtain the 
best classification accuracy. A considerable amount of research is done to find out the best classifiers, in order to detect mental disorders. In this paper, the focus is on feature extraction, selection, and classification process to obtain a highly accurate mental disease detection system. The originality of this work is in estimating performance of different algorithms, and evaluating their respective applications. This text also identifies various algorithms for feature extraction, feature selection, domain exploration, dataset combination, cross linkage between various disorders, classification, and post processing models for improving efficiency of mental disorder condition estimation. For instance, from this research it is estimated that, high end classification algorithms like CNN, LSTM-based CNN, Deep CNN and other CNN architectures are identified to be used for training and system evaluation. Similar observations are made for other techniques, and their performance is estimated.

In the next section we describe the various algorithms and systems proposed by researchers for detection of these kind of diseases and recommend improvements in the same. Light is also shed on, some variations of existing systems which will allow for higher accuracy. Finally, the paper concludes with some interesting observations about the reviewed algorithms and propose improvements in the same.

\section{LITERATURE REVIEW}

Psychiatric disorders are growing at an unprecedented rate, mainly due to the growing digital culture of the society. The work proposed in [1] provides a comprehensive review about the different kind of techniques that can be used to detect these conditions. For instance, Magnetic resonance imaging or MRI can be one of the most effective techniques to analyse a person's Brain imagery. This imagery is able to identify various components like white matter, grey matter, Brain fluid, lobe positions, etc. Based on the pattern of change of these components, medical practitioners can identify the psychic health level of the individual. Electroencephalography or EEG is another non-invasive way to evaluate the brain waves, and thereby observe the mental health of the patient under test. Kinesics of the person, that include voice data, behavioural patterns, facial patterns, social patterns, etc. are another set of psychic evaluation data for the patient under test. Generally, Kinesics data combined with the MRI and EEG scans can prove to be a good technique for evaluating the person's mental state. Algorithms like Bayesian model, Logistic regression, Decision tree, Support vector machines, and Deep learning methods like CNN [1] can be used for classifying this data. While, algorithms like Bayesian model, SVM and others have limited accuracy and precision because they are simpler to implement and have less computational complexity; CNN and other deep learning-based models will always outperform them. Since CNN models have the highest number of feature extraction units, which increases the accuracy of these systems many-folds.

The adolescent age has the highest probability of developing psychological issues. Most of these issues could not be detected until now, due to limited conversation visibility between the adolescent person and the responsible adult. Due to this, these adolescent persons later develop disorders like anxiety, abnormal social behaviour, suicidal tendency, depression, bipolar disorder, obsessive-compulsive disorder (OCD), etc. But, due to the advent of social media, this communication gap has narrowed. Now, the concerned adults can observe their child's online activities and take appropriate actions as and when needed. A study related to this is done in [2] where psycholinguistic features are combined with rule-based classifiers to find out the mental state of the person. They have used the Reddit Self-reported Depression Diagnosis dataset (RSRD3) to evaluate their proposed model. It is seen that their proposed rule-based model outperforms $\mathrm{CNN}$ and fast text methods in terms of accuracy, precision, recall, and f-measures. They claim to have achieved more than $90 \%$ accuracy for static datasets. But their work must be evaluated for dynamic and real-time datasets as well. While the work in [2] majorly focuses on detecting depression, it can be extended for other mental disorders like suicide, OCD, etc. Similar work can be seen in [3], wherein researchers have claimed to detect suicidal actions of individuals purely from their online activities. They have developed a Knowledge Ensemble and Transferring based classifiers in order to perform this task. It has been combined with a Two-step Optimization algorithm for further improvement in the classification performance. Their method claims to successfully identify suicide cases before they occur with an accuracy of more than $90 \%$ across multiple static datasets of Twitter and Reddit. This claim might be true, but it should be verified by considering real-time datasets. The major effect of these mental disorders leads to indifferent decision making by the patient. These decisions are often taken against normal social behaviour. The feeling of insecurity among such patients makes challenging to make the right decisions. Such a study is done in [4], wherein shame as a feeling has found to be deeply linked with these brain-related disorders.

Apart from social media Kinesics, a person's mental state can be studied using their speech. A large amount of research has been done in this area. Disorders like Depression, PTSD, Schizophrenia, Anxiety, Bipolar, Bulimia, Anorexia, and OCD can be detected from speech signals [5]. Out of these disorders, Depression accounts for more than $40 \%$ of research. For identifying speech-related disorders techniques like Clinician assessments using perceptual rated questionnaires, Self-assessments, and Automated computational assessments based on sensors are used. Out of these techniques, the sensor-based technique is most widely used. This is because this technique is able to identify disorders with the utmost 
accuracy. The steps needed for performing classification based on the acoustic sensors are already given in the previous section. From the previous section, its identified that feature extraction of these acoustic signals plays a very important role in the effective mental analysis. Researchers in [5] have analysed different features in order to identify these diseases. A comprehensive representation of these features can be seen from figure 1 .

From the figure, its observed that Mel frequency components and fo features are the best choice for classification of most of the mental disorders; except OCD which can be evaluated using Jitter, Shimmer and Tremor features. This complex analysis of feature extraction should help researchers to select the best features for a given mental disorder classification application. These techniques can be combined with an effective series of classifiers and post-processing techniques in order to design an effective classification system.

The work done in [6] assists this review in identifying the best classification techniques which can be used for the evaluation of mental disorders. Figure 2 showcases the different deep learning-based algorithms which are reviewed by the researchers. Using these algorithms, the system designers can design different combinations of deep learning-based classification mechanisms.

These researchers have also performed an in-depth performance evaluation of these algorithms. Most of this comparison is on MR imagery and EEG datasets, but it can be equally extended to acoustic signals as well.

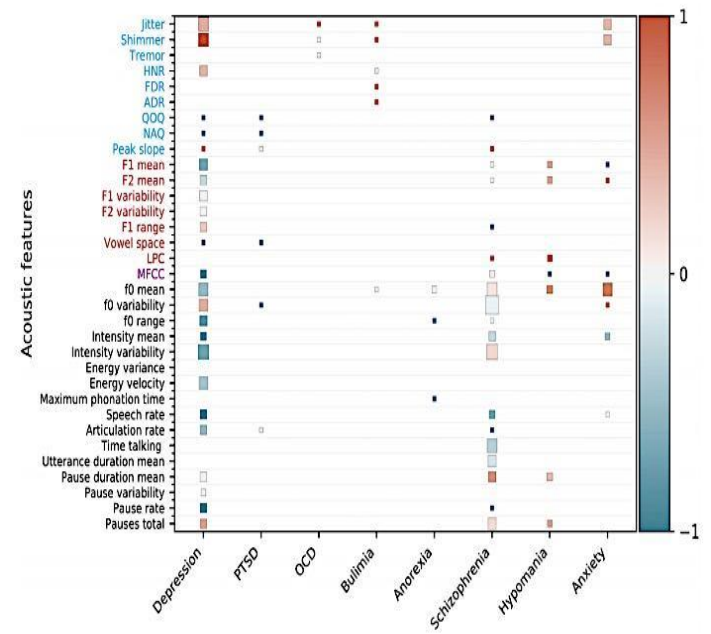

Figure 1 Analysis of acoustic feature extraction techniques for mental disorders.

ADNI, OASIS, CUH, and GODC datasets are used for the purpose of classification. Results demonstrate that Deep CNN, SAE-based CNN, and ternary CNN classifiers are the best in terms of classification accuracy across different datasets. Recommend that, the system designer should combine the features extracted in [5] with the classification methods in [6], and define a comprehensive model for acoustic features-based classification of a patient's mental health. From table 1, its observe that CNN-based algorithms are the most suited for classification across all kinds of mental disorders. The usage of these algorithms typically indicates that the most used algorithms have the highest classification accuracy.

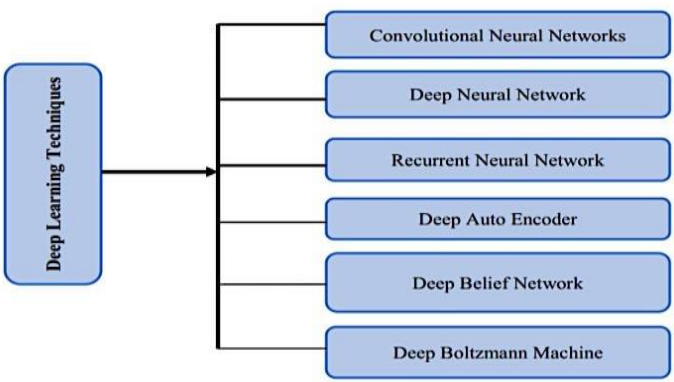

Figure 2 Different deep learning techniques.

Table 1. Algorithm usage comparison across different mental disorders.

\begin{tabular}{lcrrcccc}
\hline Disease & SAE(DA) & CNN & DNN & DBN & RNN & DBM & Other Method \\
\hline Stroke & $22 \%$ & $56 \%$ & $0 \%$ & $0 \%$ & $0 \%$ & $0 \%$ & $22 \%$ \\
Alzheimer's & $18 \%$ & $47 \%$ & $11 \%$ & $0 \%$ & $3 \%$ & $3 \%$ & $18 \%$ \\
Parkinson's & $8 \%$ & $54 \%$ & $8 \%$ & $8 \%$ & $0 \%$ & $0 \%$ & $23 \%$ \\
Epilepsy & $19 \%$ & $44 \%$ & $6 \%$ & $6 \%$ & $25 \%$ & $0 \%$ & $0 \%$ \\
Autism & $17 \%$ & $17 \%$ & $50 \%$ & $0 \%$ & $0 \%$ & $0 \%$ & $17 \%$ \\
Migraine & $0 \%$ & $100 \%$ & $0 \%$ & $0 \%$ & $0 \%$ & $0 \%$ & $0 \%$ \\
Cerebral Palsy & $100 \%$ & $0 \%$ & $0 \%$ & $0 \%$ & $0 \%$ & $0 \%$ & $0 \%$ \\
Multiple Sclerosis & $0 \%$ & $67 \%$ & $0 \%$ & $0 \%$ & $0 \%$ & $0 \%$ & $33 \%$ \\
\hline
\end{tabular}

This can again be verified from table 2, wherein algorithms are compared w.r.t their accuracies. Social media data could be used intelligently to assist in effective mental health analysis. The work done in [7] indicates that there enough sources of social media data that can easily be identified for any user. These sources include Facebook, Twitter, Amazon shopping, music, Google search history, WhatsApp, and more. In order to capture this data, the researchers have indicated a flow strategy. The strategy can be seen from figure 3 . It indicates that the user provides their own credentials, thereby there is no hacking or spying. Data is anonymized using standard partitioning and then sent for analysis.

Table 2. Algorithm comparison on Mental Disease Classification.

\begin{tabular}{llc}
\hline Disease & Methodology & Accuracy \\
\hline Stroke & SVM, SAE & $90 \%$ \\
Alzheimer's & CNN & $100 \%$ \\
Parkinson's & CNN & $98.8 \%$ \\
Epilepsy & RNN & $99.6 \%$ \\
Autism & DNN & $90.39 \%$ \\
Migraine & CNN & $99.25 \%$ \\
Multiple Sclerosis & Deep Learning & $99.78 \%$ \\
\hline
\end{tabular}


From this table, its observed that $\mathrm{CNN}$ and it's variants are the best option when it comes to classification of mental disorders. Thus, when combined with other features like acoustics, or question-answer analysis, it should produce promising results when used with an effective feature extraction layer like the one given in [5]. Anonymization is needed because the requested data is processed over the cloud, and thus will always be prone to spoofing and spying attacks at the bare minimum. Emotional features are extracted from this data and then classifiers like CNN and other machine learning variants are applied on these features. The final output is the emotional state of the person, which can further be mapped using time-series analysis to different mental state conditions.

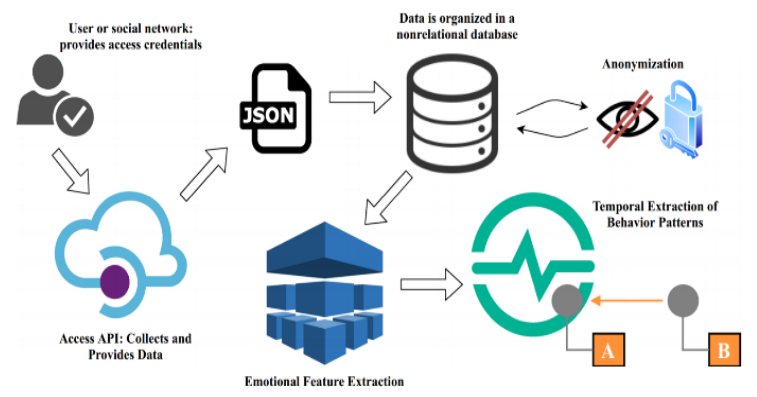

Figure 3 Data acquisition from social network for Mental State Analysis.

Machine learning algorithms like deep CNN, LSTM (Long Short-Term Memory), max pooling, BiLSTM, etc. for identifying suicidal conditions are described [8] are further categorized into Feature Engineering algorithms and pure Deep Learning algorithms. Feature engineering algorithms are largely dependent on fixed features like Word counts, POS (Parts of Speech), LIWC (Linguistic Inquiry Word Count), TF-IDF (Term-Frequency, Inverse Document Frequency), Multivariate/univariate logistic regression, screening questionnaire, Vocabulary features, syntactic features, semantic class features, N-gram, Dictionary, SVD (Singular Value Decomposition), readability score, BoWs, empath, readability, syntactic, topic, emotion and lexicon to mention a few. While pure deep learning algorithms depend upon Word embedding, tabular features, D-CNN, max pooling, Multi-task learning, neural networks, Pretrained GRU (gated recurrent units), NeuNetS, Dual-context, BERT (Bidirectional Encoder Representations from Transformers), user-factor adaptation, knowledge base, ConceptNet embedding, GloVe (global vectors for word representation), BiLSTM and self-attention to name a few. All these algorithms can work effectively on both text and acoustic data. Here too, LSTM-based encoders and CNNs are the optimum choice as given by the researchers to classify the social media data and observe the mental state of the user. The work is restricted to suicidal tendencies, but it can be extended to other disorders as well.
As stated previously, speech can be a very good tool for analysis of the mental state of a person. The flow diagram of how speech processing can be done to detect stress from acoustic signals can be seen in figure 4 . Here, the same process as indicated in the introduction is followed. From this, we can observe that feature extraction and machine learning algorithm for classification are the main blocks that require research.

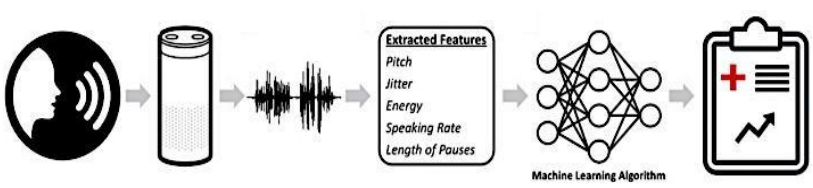

Figure 4 Processing of acoustic signals for mental state analysis.

For this, the work from [5] and [6] can be combined, wherein features can be extracted using MFCC and f0 measures, while $\mathrm{CNN}$ and other variants can be used for classification. A very strong performance evaluation of such a system is done [10], wherein a real time dataset consisting of 93 university students (45 males, 48 females) with varying levels of anxiety was taken. These students were given a speech to prepare and based on that speech analysis it was concluded whether a particular student has anxiety disorder or not. In their work, the researchers did not use any algorithm for classification, but instead performed this classification using visual analysis; which can be a good real-time dataset source for the readers of this text. Features like vocal quality, Adequacy of Gaze, Low Discomfort, and flow of speech to identify the student's mental condition is seen and also observed that the performance of the student is at par independent of whether the student has anxiety or not; but the condition is that the student must be under adequate social pressure. This work is majorly based on visually statistical comparison, which is majorly based on statistical evaluation of feature sets for mental disorder detection. In [11], different signals like EEG, ECG, EDA, EMG, respiratory rate, speech, skin temperature, pupil size and eye activity to evaluate the mental condition of the patient under test. They indicate that vocal features like Pitch, Formant 1, 2, Spectral slope, Utterance duration, Glottal pulse, Duration of words, Duration of vowel, Duration of diphthong, Intensity and Jitter in voice are enough to describe the human voice for stress evaluation. They indicate that pitch, formant 1,2, duration of words, vowels, diphthongs, voice intensity and jitter significantly increase when a person is under stress or suffering from a mental disorder, while formant 2 and Glottal pulse reduce during the same condition. They also indicate that algorithms like MFCC, TEO-CB Auto-Env, HMM, GMM, and SVM are better to process speech signals for accessing the patient's mental state. It's stated that, accuracy levels up-to $90 \%$ can be achieved with the help of these algorithms. While speech utterance can be a tool to identify mental disorders, speech hearing impairment is another cause of anxiety 
among adolescents. The work in [12] indicates this and concludes that people with hearing disabilities have a high probability of anxiety between $7 \%$ to $39 \%$ or more. In contrast to this, the work done in [13] extends the work done in [11] and showcases that mobile device can be used as an invaluable tool to detect mental conditions. They tested their algorithm DAIC-WOZ dataset and observed that the real-time accuracy is very low (around 50\%), and thereby it needs to be improved significantly. The architecture for their model is shown in figure 5 .

From this its observed that there is a large scope of improvement in speech analysis for real-time datasets, and it can be taken up as a research problem. This work can be extended by adding other non-invasive feature extraction techniques like EEG classification. This can be seen from [14], wherein EEG has been used as a feature vector for the DASPS dataset.

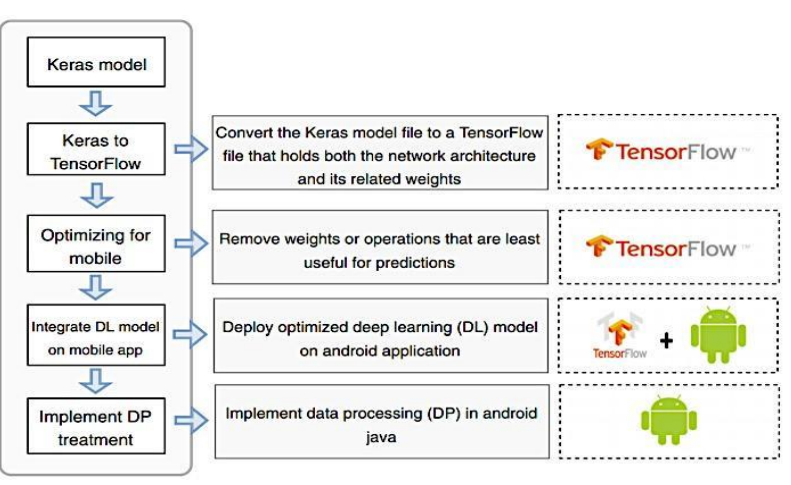

Figure 5 Mobile processing for mental disorders.

This dataset consists of 23 participants during anxiety conditions under face-to-face encounters. They have used auto-encoders due to which the overall accuracy of the system goes up to $83 \%$ and can be further extended to $90 \%$ with the help of neural nets. Their architecture combines algorithms like SVM, KNN and SSAE (Stacked Sparse Auto-Encoders) to develop a hybrid and highly effective system. They have also proposed the use of fractal dimensions, Hjorth parameters, Hilbert-Huang spectrum, FFT-based band power, wavelet transform and its variants for evaluating features from the EEG signals. As EEG and acoustic signals are both one dimensional in nature, the same algorithm set can be directly applied to acoustic signals as well. The evaluation of such a system is yet to be done.

Another comprehensive review on mental disorder detection using non-verbal communication methods is mentioned in [15]. Here researchers have taken features like Pupil dilation / bias, Pupillary response, Iris movement Eyelid activity, (opening, blinking) Saccadic eye movements, Eye gaze (limited \& shorter eye contact), Visual fixation, Low frequency \& duration of glances, Extended activity on the corrugator muscle, Eyebrow activity, Veraguth fold, Frowns, Fewer smiles, More frequent lip presses, Smile intensity \& duration, Mouth corners angled down, Mouth animation, Listening smiles (smiling while not speaking), Reduced activity on the zygomaticus, Facial activity, Action Units occurrence (mean duration, ratio of \{ onset / total duration, onset / offset\}), Region Units (RUs), Facial expression occurrence (variability \& intensity), Sad / negative / neutral expression occurrence, Head pose (orientation, movement), Body gestures (full or upper body, or body parts), Slumped posture Limp \& uniform body posture, Reduced \& slowed arm and hand movements, Shaking and/or fidgeting behaviour, Self-adaptors, Foot tapping and Motor variability. Based on these parameters the user's mental state can be evaluated. The workflow for such an assessment can be visualized from figure 6 as follows:

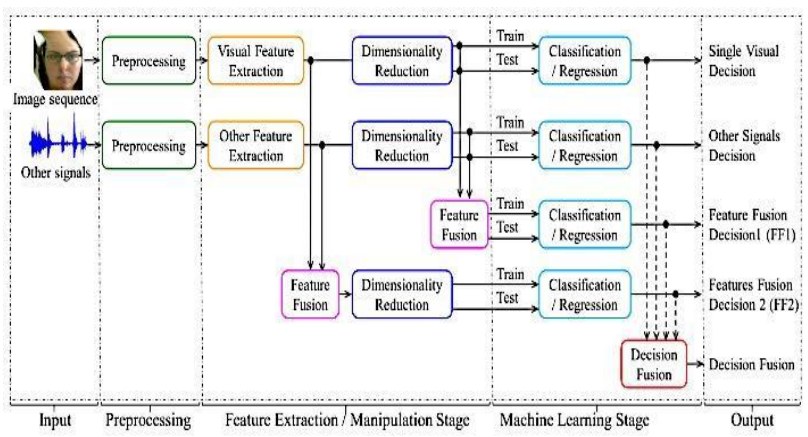

Figure 6 Workflow of the visual analysis system.

It's observed that pre-processing, feature extraction, feature reduction, classification and post-processing are the steps in any kind of classification system, and mental disorder detection system also falls into the fact. Algorithms like SVM and Logistic regression outperform other algorithms in terms of accuracy. While this work doesn't take into consideration the performance analysis using CNNs, that can be done, and the results can be validated. The most widely used data classification algorithm is CNN. This can be seen from [16], wherein CNNs are used for speech-based screening of depression. In their work, researchers have taken real-time inputs from more than 2000 individuals, and then evaluated their mental state. Features like wavelet, spectrogram along with data augmentation were used in order to process the sound samples. Based on their 8 layered model, an accuracy of more than $80 \%$ was observed. This is a very high value considering that real-time datasets were used. This can further be extended by using features like MFCC, F0, F1, F2, etc. and then combining them with wavelet and spectrogram features. While the work is focused on depression analysis, with proper training it can be used for any kind of mental state analysis. While sound is the rhythm of the mouth, the ECG is the rhythm of the heart. Whenever a person is depressed or is facing a mental state disorder, then there are variations in this rhythm. The work in [17] proposes a novel feature named as range of local Hurst exponents (RLHE) to identify the relation between the heart rhythm of a person with a healthy mood, and a 
person with a non-healthy condition. This novel feature can boost up the accuracy of classification to $85 \%$, while the true negative rate is boosted to $97 \%$. The researchers have used SVM as their choice of classifier, which is combined with RLHE and statistical heart rate features. The work majorly focusses on anxiety detection but can be extended for any kind of mental disorder condition. Moreover, the RLHE feature set can be applied to acoustic signals, and SVM can be replaced by AI methodologies to boost the classification accuracy. As per approximations, the accuracy can be boosted up-to $90 \%$ to $95 \%$ based on the choice of acoustic dataset. In order to do so, the work in [18] can be taken up as a reference. In this work, algorithms like Random forests, neural networks, and elastic nets are studied and their accuracy is evaluated for understanding human mental conditions. From their research it is evident that fusion of CNN methods \& identification of predictive features can boost the accuracy of classification to more than $95 \%$. But this claim is not supported by any statistical analysis, and thus researchers must perform due diligence before using such a system. Meta-analysis [19] can be another approach to identify mental disorders among adolescents. The work claims that meta-analysis performed on a group of persons can be useful in analysing the overall trend of mental disorders like anxiety disorders, social phobia, separation anxiety disorder, specific phobia, generalized anxiety disorder and obsessive-compulsive disorder. Other methods of stress detection might include Heart Rate Variability (HRV) analysis, humidity response and Temperature response as indicated in [20]. The HRV analysis is majorly based on ECG analysis as mentioned previously, while temperature and humidity responses are majorly based on trial-anderror methods. These methods can be combined with speech in order to improve the accuracy of mental disorder detection. A summary of these body parameters and their effect on the person's mental state can be observed from table 3. It's indicated that higher Heart rate, blood pressure, GSR, humidity and temperature values will always result in a stressed mindset, and other observations follow this. Thus, while performing speech-based analysis; if these parameters are taken into consideration, then the overall accuracy might improve significantly.

Table 3. Body parameter variation on the mental state of a person.

\begin{tabular}{|c|c|c|c|c|}
\hline \multirow{2}{*}{ Condition } & \multicolumn{4}{|c|}{ Parameters } \\
\cline { 2 - 5 } & HR & BP & GSR & H\&T \\
\hline Stressed & $>100$ & $\begin{array}{c}\text { bps }>130 \\
\text { bps }>110\end{array}$ & $>6$ & $<33$ \\
\hline Tensed & $90-100$ & $\begin{array}{c}120 / 90- \\
130 / 110\end{array}$ & $4-7$ & $33-35$ \\
\hline Calm & $70-90$ & $\begin{array}{c}110 / 75- \\
120 / 85\end{array}$ & $2-4$ & $35-36$ \\
\hline Relaxed & $60-70$ & $\begin{array}{c}100 / 70- \\
110 / 75\end{array}$ & $<2$ & $36-37$ \\
\hline
\end{tabular}

Increasing the valid number of features increases the accuracy of classification, this can be proven from the work in [21], where researchers have introduced features like PC1, PC6, PC17 and PC24 for voice-based mental state analysis. Using these features the accuracy of classification was boosted by more than $36 \%$. They have used a simple classifier namely Binary logistic regression for this purpose. The usage of LSTM-based CNN and a combination of MFCC, and PC features in order to identify different mental disorders is recommended. This would definitely improve the performance of the system under test. Such a system would require a large amount of data for training and validation. The work in [22] provides such a dataset. Researchers have reviewed different techniques for automated screening of distress. The work has collected a large number of datasets for comparison. As the major aim of this text is to study methods related to speech, thus its advised to the readers to visit the following datasets for their system designs, AD80, French Adapted Speech Corpus, CIRDO Corpus, Distress Analysis Interview Corpus, Virtual Human Distress Assessment Interview Corpus, Distress Analysis Interview Corpus-Wizard of Oz, Audio-Visual Depressive Language (AViD) Corpus, AVEC 2014 Audio-Visual Depression Corpus (AVEC), Black Dog, Pittsburgh and ORYGEN. All these datasets combined would accumulate to form a large enough training corpus, that can be used to train an effective LSTM-based CNN. Such a network will be able to obtain high levels of accuracy with minimum error rates.

Suicides are becoming more common these days. In recent days many Bollywood stars, Business peoples are committing suicide due to depression. The work in [23] analyses platforms like Reddit Suicide Watch and Twitter in order to analyse a person's profile \& suggest the probability of suicide. They analysed that a person uses Personal nouns, Anxiety, Sadness, Negative emotions, Present focus, Work and Death when he/she has suicidal tendencies. While for non-suicidal cases, Quantifiers, Positive emotion, references to gender, etc. are majorly used. For feature extraction purpose, Statistical Features, Syntactic Features, Linguistic Features, Word Frequency Features, Word Embedding Features and Topic Features were used. While algorithms like SVM, Random Forest, GBDT, XGBoost, MLFFNN \& LSTM were used for the purpose of classification. It is found that features like Statistics, topic, TF-IDF, POS, LIWC combined with XGBoost classifier \& word2vec word embedding with LSTM are the methods with highest accuracy of more than $95 \%$. This accuracy is for text classification, but can be extended for any kind of signal processing with proper modifications. Novel methods like Deep Brain Simulations (DBS) [24] have proven to be useful for OCD but, the after effects of such treatments are not productive. Due to this, the after effects of DBS on OCD have been analysed and, found that DBS and other brain related treatments normalizes the life of the patient, but the patient is posed with new challenges like finding difficulty to 
handle anxiety situation which arises in due course of life. These questions are currently open for research and can be opted by readers. Readers can also research speech-based methods for evaluation of post-treatment conditions of the patients who have undergone such treatments. Symptoms like psychotic and schizotypal are inherent in patients with OCD, this study is done in [25]. It also suggests methods to reduce such symptoms, like Exposure and Response Prevention (ERP). Based on this, researchers can evaluate other inter-related brain diseases as discussed in [26]. A classification system could be designed to leverage this information in order to predict the presence of mental disorders. For instance, if a person is showing psychotic and schizotypal symptoms together, then the researchers can conclude that the person has high probability of OCD as suggested in [27-30]. Such observations when combined with speech processing systems will be helpful in designing more complex classification systems. Speechbased classification systems like the one mentioned in [3036] can then be used to identify other mental disorders based on input feature variance. For example, Fundamental frequency contours, its variability and Mean articulation rate in syllables results in anger, sorrow and fear stress conditions. Conditions like these when combined with advanced classifiers would result in a highly effective mental disorder classification system. [3642]

\section{OBSERVATIONS}

Speech processing when combined with other fields of study can enhance the efficiency of mental disorder detection. From this review, the observations are, Crossdomain statistical feature extraction techniques like MFCC, F0, F1, range of local Hurst exponents (RLHE), wavelet and others significantly increase the accuracy of classification. Speech to text analysis using Statistics, topic, TF-IDF, POS, LIWC combined with XGBoost classifier \& word2vec word embedding with LSTM are the most reliable techniques for mental disorder classification. Social media analysis can be a great addition when used along with speech processing, techniques like Knowledge Ensemble and Transferring based classifiers must be used for an effective system design. Combination of multiple datasets like AD80, French Adapted Speech Corpus, CIRDO Corpus, Distress Analysis Interview Corpus, Virtual Human Distress Assessment Interview Corpus, Distress Analysis Interview Corpus-Wizard of Oz, AudioVisual Depressive Language (AViD) Corpus, AVEC 2014 Audio-Visual Depression Corpus (AVEC), Black Dog, Pittsburgh and ORYGEN must be performed in order to create a large enough trainable dataset. Algorithms like CNN, MFCC, predictive modelling, RLHE when combined with a sufficiently large number of data samples can lead to the development of an effective solution for mental disorder classification. Based on this theoretical evaluation, statistical values for accuracy of these models are observed and tabulated in table 4 , wherein different models are differentiated.

Table 4. Statistical Analysis of different algorithms

\begin{tabular}{|l|l|l|}
\hline Model & Method & Accuracy (\%) \\
\hline Bayesian model [1] & Kinesics data combined with the MRI and EEG scans & 80 \\
\hline Logistic regression [1] & Kinesics data combined with the MRI and EEG scans & 75 \\
\hline Decision tree [1] & Kinesics data combined with the MRI and EEG scans & 79 \\
\hline Support vector machines [1] & Kinesics data combined with the MRI and EEG scans & 85 \\
\hline CNN [1] & Kinesics data combined with the MRI and EEG scans & 94 \\
\hline Rule based classifier [2] & $\begin{array}{l}\text { Reddit Self-reported Depression Diagnosis dataset with } \\
\text { psycholinguistic features }\end{array}$ & 76 \\
\hline $\begin{array}{l}\text { Knowledge Ensemble and Transferring } \\
\text { based classifiers [3] }\end{array}$ & Transfer learning on multiple datasets & 90 \\
\hline Linear classifiers [5] & $\begin{array}{l}\text { Speech features for Depression, PTSD, Schizophrenia, Anxiety, } \\
\text { Bipolar, Bulimia, Anorexia and OCD detection }\end{array}$ & 85 \\
\hline CNN [6] & $\begin{array}{l}\text { Stroke, AD, Parkinson, Epilepsy, Autism, Migraine, and Cerebral } \\
\text { Palsy }\end{array}$ & 93 \\
\hline DNN [6] & $\begin{array}{l}\text { Stroke, AD, Parkinson, Epilepsy, Autism, Migraine, and Cerebral } \\
\text { Palsy }\end{array}$ & 95 \\
\hline DBN [6] & $\begin{array}{l}\text { Stroke, AD, Parkinson, Epilepsy, Autism, Migraine, and Cerebral } \\
\text { Palsy }\end{array}$ & 94 \\
\hline RNN [6] & $\begin{array}{l}\text { Stroke, AD, Parkinson, Epilepsy, Autism, Migraine, and Cerebral } \\
\text { Palsy }\end{array}$ & 91 \\
\hline DBM [6] & $\begin{array}{l}\text { Stroke, AD, Parkinson, Epilepsy, Autism, Migraine, and Cerebral } \\
\text { Palsy }\end{array}$ & 93.5 \\
\hline CNN [7] & Facebook, Twitter, and social data & 96 \\
\hline LSTM [8] & $\begin{array}{l}\text { Word counts, POS (Parts of Speech), LIWC (Linguistic inquiry word } \\
\text { count), TF-IDF (term-frequency, inverse document frequency), etc. }\end{array}$ & 92.5 \\
\hline CNN [9] & Pitch, jitter, energy, speech rate, and pause length & 94.5 \\
\hline DNN [1] & $\begin{array}{l}\text { EEG, ECG, EDA, EMG, respiratory rate, speech, skin temperature, } \\
\text { pupil size and eye activity }\end{array}$ & 96.3 \\
\hline
\end{tabular}




\begin{tabular}{|l|l|l|}
\hline GMM [11] & $\begin{array}{l}\text { EEG, ECG, EDA, EMG, respiratory rate, speech, skin temperature, } \\
\text { pupil size and eye activity }\end{array}$ & 97.2 \\
\hline Auto Encoder [14] & EEG for signal processing & 83 \\
\hline CNN [14] & EEG for signal processing & 90 \\
\hline Ensemble classification [15] & SVM, kNN and SSAE (Stacked Sparse Auto-Encoders) & 91 \\
\hline SVM [16] & MFCC, F0, F1, F2 & 85 \\
\hline LR [16] & MFCC, F0, F1, F2 & 90 \\
\hline RLHE [17] & SVM combined with RLHE & 85 \\
\hline RF [18] & Acoustic features & 80 \\
\hline Elastic Nets [18] & Acoustic features & 85 \\
\hline CNN [18] & Acoustic features & 91 \\
\hline CNN [21] & Heart rate variability & 96 \\
\hline LSTM [23] & XGBoost classifier \& word2vec word embedding & 95 \\
\hline DBS [24] & CNN for OCD detection & 91 \\
\hline
\end{tabular}

From this result, it is observed that deep learning models outperform other models in terms of classification accuracy for various types of diseases. This can also be observed from figure 7, wherein different algorithms, and their accuracies are compared for visual reference.

\section{Accuracy of different models (\%)}

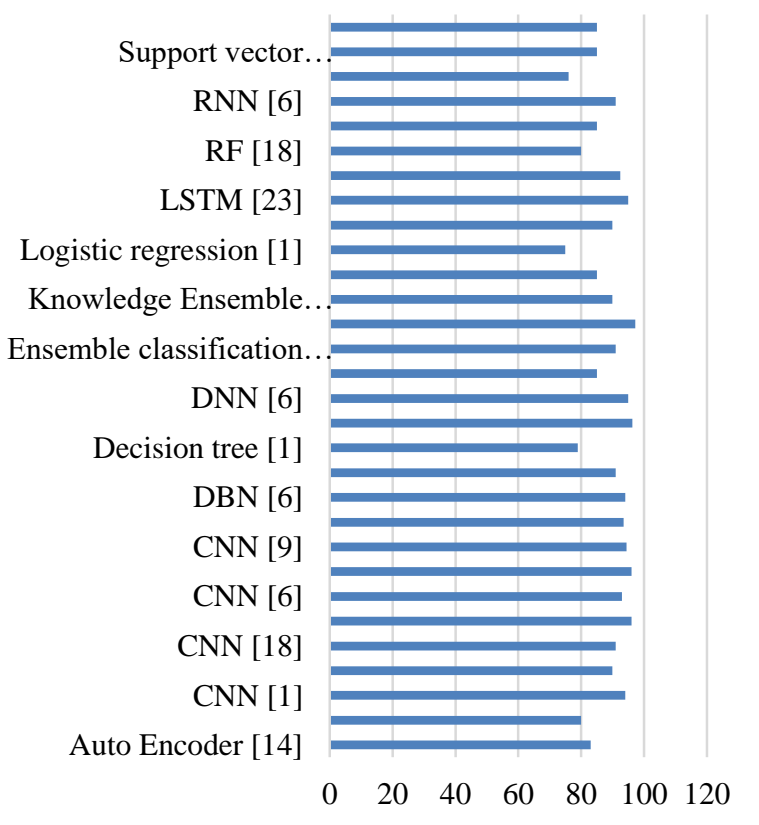

Figure 7 Taxonomy of different algorithms

From this taxonomy, it can be observed that GMM [11], DNN [11], CNN [7], CNN [21], DNN [6], LSTM [23], CNN [9], CNN [1] and DBN [6] outperform other models for mental health analysis, and thus must be used by researchers and system designers for high performance applications.

\section{CONCLUSION FUTURE WORK}

Cross-linkage between different disorders needs to be considered to maximize the probability of disease detection. For instance, a person showcasing psychotic and schizotypal symptoms together, might have high probability of OCD. EEG, ECG, Temperature, Humidity, and other body parameters can be combined with speech input in order to further optimize the accuracy of mental state detection. High end classification algorithms like CNN, LSTM-based CNN, Deep CNN and other CNN architectures must be used for the purpose of training and system evaluation. Post treatment mental disorder analysis is very important for patients to lead a normal life. Prediction of future behaviour using predictive modelling must be done in order to perform post-processing of mental conditions. Based on these observations its observed that, there are many open issues prevalent to mental disorder detection. Issues like cross-linkage of disorders, combination of social media data with speech data, etc. need to be solved in order to develop an effective system.

\section{REFERENCES}

[1] Guang-Di Liu, Yu-Chen Li, Wei Zhang, Le Zhang. A Brief Review of Artificial Intelligence Applications and Algorithms for Psychiatric Disorders. 2020, 462467.

[2] Alina Trifan, Rui Antunes, S'ergio Matos, and Jose Luis Oliveira. Understanding Depression from Psycholinguistic Patterns in Social Media Texts.

[3] Shaoxiong Ji. Suicidal Ideation Detection in Online Social Content.

[4] Aurora Szentágotai-Tătar, Diana-Mirela Nechita, Andrei C. Miu. Shame in Anxiety and ObsessiveCompulsive Disorders. 2020, 10.1007/s11920-0201142-9.

[5] Daniel M. Low, Kate H. Bentley, Satrajit S. Ghosh. Automated assessment of psychiatric disorders using speech: A systematic review. 17 January 2020, 10.1002/lio2.354.

[6] Ritu Gautam, Manik Sharma. Prevalence and Diagnosis of Neurological Disorders Using Different Deep Learning Techniques: A Meta-Analysis. 2020, 10.1007/s10916-019-1519-7.

[7] Felipe T. Giuntini1 · Mirela T. Cazzolato1 · Maria de Jesus Dutra dos Reis2. Andrew T. Campbell3. Agma 
J. M. Traina1. Jó Ueyama. A review on recognizing depression in social networks: challenges and opportunities. 2020, 10.1007/s12652-020-01726-4.

[8] Shaoxiong Ji, Student Member, IEEE, Shirui Pan, Member, IEEE, Xue Li, Erik Cambria, Senior Member, IEEE, Guodong Long, and Zi Huang. Suicidal Ideation Detection: A Review of Machine Learning Methods and Applications. 23 oct2019.

[9] George M. Slavich, Sara Taylor \& Rosalind W. Picard. Stress measurement using speech: Recent advancements, validation issues, and ethical and privacy considerations. 04 Apr 2019, ISSN: 10253890 1607-8888.

[10] Trevor Thompson, Nejra Van Zalk, Christopher Marshall, Melanie Sargeant and Brendon Stubbs. Social anxiety increases visible anxiety signs during social encounters but does not impair performance. 2019, 10.1186/s40359-019-0300-5.

[11] Giorgos Giannakakis,Member, IEEE, Dimitris Grigoriadis, Katerina Giannakaki, Olympia Simantiraki, Alexandros Roniotis andManolis Tsiknakis, Member, IEEE. Review on psychological stress detection using biosignals.

[12] Natalie Shoham, Gemma Lewis, Graziella Favarato, Claudia Cooper. Prevalence of anxiety disorders and symptoms in people with hearing impairment: a systematic review. 2019 54:649-660.

[13] Afef Mdhaffar, Fedi Cherif, Yousri Kessentini, Manel Maalej, Jihen Ben Thabet, Mohamed Maalej, Mohamed Jmaiel, and Bernd Freisleben. DL4DED: Deep Learning for Depressive Episode Detection on Mobile Devices.

[14] Asma Baghdadi, Member, IEEE, Yassine Aribi, Senior Member, IEEE, Rahma Fourati, Member, IEEE, Najla Halouani, Patrick Siarry, Senior Member, IEEE and Adel M. Alimi, Senior Member, IEEE. DASPS: A Database for Anxious States based on a Psychological Stimulation. 5 aug 2019.

[15] Anastasia Pampouchidou, Panagiotis Simos, Kostas Marias, Fabrice Meriaudeau, Fan Yang, Matthew Pediaditis, and Manolis Tsiknakis. Automatic Assessment of Depression Based on Visual Cues: A Systematic Review. DOI: 10.1109/TAFFC.2017.2724035.

[16] Karol Chlastaa,b*, Krzysztof Wołka, Izabela Krejtz. Automated speech-based screening of depression using deep convolutional neural networks. 2019.

[17] Wanhui Wen, Guangyuan Liu, Zhi-Hong Mao, Wenjin Huang, Xu Zhang, Hui Hu, Jiemin Yang and Wenyan Jia. Toward Constructing a Real-time Social Anxiety Evaluation System: Exploring Effective
Heart Rate Features. DOI 10.1109/TAFFC.2018.2792000.

[18] Mihaela Robila, Stefan A. Robila. Applications of Artificial Intelligence Methodologies to Behavioral and Social Sciences. 2019 10.1007/s10826-01901689-x.

[19] Jim Driessen, Jan Dirk Blom, Peter Muris, Roger K. Blashfield, Marc L. Molendijk. Anxiety in Children with Selective Mutism: A Meta-analysis. 2019 10.1007/s10578-019-00933-1.

[20] G. Shanmugasundaram, S.Yazhini, Hemapratha .E, Nithya .S. A Comprehensive Review on Stress Detection Techniques. 2019, 978-1-7281-1524-5.

[21] Wei Pan, Jonathan Flint, Liat Shenhav, Tianli Liu, Mingming Liu1, Bin $\mathrm{Hu}$, Tingshao Zhu. Reexamining the robustness of voice features in predicting depression: Compared with baseline of confounders. June 20, 2019.

[22] Rajib Rana, Siddique Latif, Raj Gururajan, Anthony Gray, Geraldine Mackenzie, Gerald Humphris, and Jeff Dunn. Automated Screening for Distress: A Perspective for the Future. 22 feb 2019.

[23] Shaoxiong Ji, Celina Ping Yu, Sai-fu Fung, Shirui Pan , and Guodong Long. Supervised Learning for Suicidal Ideation Detection in Online User Content. Volume 2018, Article ID 6157249.

[24] Peter Bosanac, Bridget Elizabeth Hamilton, James Lucak and David Castle. Identity challenges and 'burden of normality' after DBS for severe OCD: a narrative case study. 2018, 10.1186/s12888-0181771-2.

[25] Stian Solem1*, Kristen Hagen2, Christoffer Wenaas1, Åshild T. Håland3, Gunvor Launes3, Patrick A. Vogel1, Bjarne Hansen4 and Joseph A. Himle. Psychotic and schizotypal symptoms in nonpsychotic patients with obsessive-compulsive disorder. 2015, DOI 10.1186/s12888-015-0502-1.

[26] Muhammad Sharif, Javaria Amin, Mudassar Raza, Muhammad Almas Anjum, Humaira Afzal, Shafqat Ali Shad. Brain tumor detection based on extreme learning. 11 January 2020,doi.org/10.1007/s00521019-04679-8.(0.

[27] Chang Su, Zhenxing Xu, Jyotishman Pathak and Fei Wang. Deep learning in mental health outcome research: a scoping review. 2020, /10.1038/s41398020-0780-3. ().,-[28] Marcin Moskalewicz1 \& Michael A. Schwartz. Temporal experience as a core quality in mental disorders. 12 march 2020, 10.1007/s11097-020-09665-3. 
[29] K. Yu, L. Tan, L. Lin, X. Cheng, Z. Yi and T. Sato, "Deep-Learning-Empowered Breast Cancer Auxiliary Diagnosis for 5GB Remote E-Health," IEEE Wireless Communications, vol. 28, no. 3, pp. 54-61, June 2021, doi: 10.1109/MWC.001.2000374.

[30] K. Yu, L. Tan, S. Mumtaz, S. Al-Rubaye, A. AlDulaimi, A. K. Bashir, F. A. Khan, "Securing Critical Infrastructures: Deep Learning-based Threat Detection in the IIoT", IEEE Communications Magazine, 2021.

[31] K. Yu, Z. Guo, Y. Shen, W. Wang, J. C. Lin, T. Sato, "Secure Artificial Intelligence of Things for Implicit Group Recommendations", IEEE Internet of Things Journal, 2021, doi: 10.1109/JIOT.2021.3079574.

[32] H. Li, K. Yu, B. Liu, C. Feng, Z. Qin and G. Srivastava, "An Efficient Ciphertext-Policy Weighted Attribute-Based Encryption for the Internet of Health Things," IEEE Journal of Biomedical and Health Informatics, 2021, doi: 10.1109/JBHI.2021.3075995.

[33] L. Zhen, Y. Zhang, K. Yu, N. Kumar, A. Barnawi and Y. Xie, "Early Collision Detection for Massive Random Access in Satellite-Based Internet of Things," IEEE Transactions on Vehicular Technology, vol. 70, no. 5, pp. 5184-5189, May 2021, doi: 10.1109/TVT.2021.3076015.

[34] L. Tan, K. Yu, F. Ming, X. Cheng, G. Srivastava, "Secure and Resilient Artificial Intelligence of Things: a HoneyNet Approach for Threat Detection and Situational Awareness", IEEE Consumer Electronics Magazine, 2021, doi: 10.1109/MCE.2021.3081874.

[35] Puttamadappa, C., and B. D. Parameshachari. "Demand side management of small scale loads in a smart grid using glow-worm swarm optimization technique." Microprocessors and Microsystems 71 (2019): 102886.

[36] Rajendrakumar, Shiny, and V. K. Parvati. "Automation of irrigation system through embedded computing technology." In Proceedings of the 3rd International Conference on Cryptography, Security and Privacy, pp. 289-293. 2019.

[37] Subramani, Prabu, K. Srinivas, R. Sujatha, and B. D. Parameshachari. "Prediction of muscular paralysis disease based on hybrid feature extraction with machine learning technique for COVID-19 and postCOVID-19 patients." Personal and Ubiquitous Computing (2021): 1-14.

[38] Hu, Liwen, Ngoc-Tu Nguyen, Wenjin Tao, Ming C. Leu, Xiaoqing Frank Liu, Md Rakib Shahriar, and SM Nahian Al Sunny. "Modeling of cloud-based digital twins for smart manufacturing with MT connect." Procedia manufacturing 26 (2018): 1193 1203.

[39] Hu, Liwen, Ngoc-Tu Nguyen, Wenjin Tao, Ming C. Leu, Xiaoqing Frank Liu, Md Rakib Shahriar, and SM Nahian Al Sunny. "Modeling of cloud-based digital twins for smart manufacturing with MT connect." Procedia manufacturing 26 (2018): 1193 1203.

[40] Seyhan, Kübra, Tu N. Nguyen, Sedat Akleylek, Korhan Cengiz, and SK Hafizul Islam. "Bi-GISIS KE: Modified key exchange protocol with reusable keys for IoT security." Journal of Information Security and Applications 58 (2021): 102788.

[41] Arun, M., E. Baraneetharan, A. Kanchana, and S. Prabu. "Detection and monitoring of the asymptotic COVID-19 patients using IoT devices and sensors." International Journal of Pervasive Computing and Communications (2020).

[42] Kumar, M. Keerthi, B. D. Parameshachari, S. Prabu, and Silvia liberata Ullo. "Comparative Analysis to Identify Efficient Technique for Interfacing BCI System." In IOP Conference Series: Materials Science and Engineering, vol. 925, no. 1, p. 012062. IOP Publishing, 2020. 